\title{
COVID-19 et lésions myocardiques
}

\author{
Bryce Alexander MD, Adrian Baranchuk MD
}

- Citation : CMAJ 2020 July 13;192:E812-3. doi : 10.1503/cmaj.201230-f; diffusion hâtive le 25 juin 2020

Voir la version anglaise ici : www.cmaj.ca/lookup/doi/10.1503/cmaj.201230; voir la recherche connexe (en anglais) ici : www.cmaj.ca/ lookup/doi/10.1503/cmaj.200879

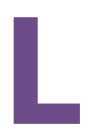

es lésions myocardiques sont fréquentes chez les patients hospitalisés; on estime qu'elles sont présentes chez $8 \%$ à $28 \%$ des patients hospitalisés en raison de la maladie à coronavirus 2019 (COVID-19) ${ }^{1}$. Définies par une élévation du taux de troponine au-delà du $99^{\mathrm{e}}$ percentile d'une population de référence saine, les lésions myocardiques associées à la COVID-19 peuvent être dues à des atteintes myocardiques, ischémiques ou non ${ }^{2}$. Les patients atteints de la COVID19 aux soins intensifs présentent une élévation des troponines plus importante que ceux hospitalisés hors des soins intensifs; des études observationnelles ont rapporté une élévation des troponines pouvant atteindre $59 \%$ chez des patients qui sont subséquemment décédés².

Dans une étude sur le même sujet, Si et ses collègues ${ }^{3}$ ont rapporté une association entre le pic de troponine et le besoin d'assistance ventilatoire et le décès chez des patients atteints de la COVID-19 admis dans un hôpital de Wuhan (Chine). Les auteurs ont conclu qu'une augmentation du pic de troponine durant l'hospitalisation était un facteur prédictif indépendant du besoin d'assistance ventilatoire effractive et de la mortalité, toutes causes confondues. Une corrélation significative a été établie entre la détection de lésions myocardiques dans les 72 heures suivant l'hospitalisation et une mortalité 10 fois plus élevée. De plus, contrairement aux autres coronavirus infectant les humains, le virus causant la COVID-19 a été associé à un nombre important d'épisodes d'arythmie, y compris des arythmies fatales.

Bien qu'il soit utile pour la communauté scientifique de montrer que les lésions myocardiques sont un facteur prédictif d'issues négatives chez les personnes atteintes de la COVID-19, il ne s'agit pas forcément d'une observation spécifique à cette maladie. La relation entre l'élévation des troponines et l'augmentation de la mortalité a été décrite précédemment dans des populations de patients hospitalisés, en soins intensifs et hors soins intensifs. Dans une étude de 2015 portant sur des patients hors soins intensifs présentant une élévation des troponines sans qu'on envisage un syndrome coronarien aigu comme premier diagnostic, on a observé une association indépendante entre l'élévation des troponines et la mortalité toutes causes

\section{POINTS CLÉS}

- Les lésions myocardiques sont fréquentes chez les patients hospitalisés qui sont traités pour la maladie à coronavirus 2019 (COVID-19), et elles ont été associées à des issues négatives dans une étude connexe.

- Il a été montré précédemment que les lésions myocardiques sont un facteur prédictif d'issues négatives dans d'autres populations de patients aux soins intensifs et pourraient donc ne pas être spécifiques à la COVID-19.

- La survenue d'arythmies semble plus fréquente chez les patients atteints de la COVID-19 que chez les personnes ayant contracté une autre infection à coronavirus; cela dit, cette différence pourrait être due au fait que les études précédentes employaient des techniques d'évaluation différentes.

- Il faudra mener d'autres recherches pour mieux caractériser la relation entre les lésions myocardiques et les issues négatives chez les patients atteints de la COVID-19.

confondues durant l'année suivante (rapport de cotes [RC] 3,37, intervalle de confiance [IC] $95 \% 1,55-7,34)^{4}$.

Il a également été montré que l'élévation des troponines est un facteur prédictif de mortalité chez les patients aux soins intensifs. Parmi les études sur le sujet, une vaste étude de cohorte rétrospective portant sur près de 20000 patients d'unités mixtes de soins intensifs qui avait utilisé les antécédents de syndrome coronarien aigu comme critère d'exclusion a montré que l'élévation des troponines était un facteur prédictif indépendant de mortalité dans les 30 jours, après ajustement pour tenir compte de la gravité de la maladie (RC 1,82, IC $95 \%$ $1,62-2,04)^{5}$. Chez les patients atteints de sepsis, une métaanalyse ambitieuse regroupant 13 études et portant sur 1227 patients admis aux soins intensifs a montré qu'une élévation des troponines durant le premier jour d'hospitalisation était un facteur de risque de décès indépendant ${ }^{6}$. L'élévation des troponines pourrait potentiellement servir de marqueur de la gravité de l'atteinte, ce qui n'est pas spécifique à la COVID-19 et pourrait être utilisé pour la stratification des risques. 
L'élévation de la troponine cardiaque hypersensible est un paramètre de laboratoire couramment détecté chez les patients se présentant à l'hôpital. Une étude prospective de 2019 a conclu que la prévalence de l'élévation des troponines au-delà du seuil du $99^{e}$ percentile dans une population se présentant aux urgences sans soupçon de syndrome coronarien aigu était de $12,4 \%{ }^{7}$. La fréquence d'élévation des troponines chez les patients aux soins intensifs était encore plus élevée. Une revue systématique de 2008 portant sur 20 études et 3278 patients a montré une prévalence de $43 \%{ }^{8}$. Dans le cas des patients admis aux soins intensifs avec un diagnostic de sepsis ou de choc septique, la prévalence de l'élévation des troponines atteignait même $60 \%{ }^{8}$. Le fait qu'une étude liée ${ }^{3}$ n'ait rapporté une élévation des troponines que chez $14,7 \%$ des patients pourrait simplement découler de ce que les patients du groupe sans élévation étaient moins gravement malades, ce qui pourrait expliquer en partie les différences extrêmes concernant la mortalité.

$\mathrm{Si}$ et ses collègues ont noté l'incidence particulièrement élevée d'arythmies observée dans le cadre de leur étude, comparativement à une étude précédente portant sur des patients atteints du syndrome respiratoire aigu sévère (SRAS), durant laquelle aucune arythmie significative sur le plan hémodynamique n'avait été observée ${ }^{9}$. Cela dit, les patients ayant participé à l'étude sur le SRAS n'ont pas été suivis à l'aide d'un monitorage cardiaque en continu, contrairement aux patients de l'étude plus récente. Ils ont plutôt subi un électrocardiogramme chaque fois qu'il était indiqué sur le plan clinique (tachycardie, bradycardie, hypotension ou autre indication). Il est plausible que l'incidence supérieure d'arythmies observée dans l'étude récente découle de l'efficacité accrue de la détection par le monitorage en continu. Bien sûr, il n'est pas rassurant que 6 patients aient subi une arythmie fatale, mais l'absence d'une population témoin ne permet pas d'en attribuer la cause à la COVID-19, puisque la gravité de l'état des patients à l'étude aurait pu jouer un rôle dans le décès. Enfin, les auteurs ont inclus des données sur l'allongement de l'intervalle QT dans les cas où les patients prenaient des médicaments ayant cet effet. La formule utilisée pour calculer l'intervalle QT corrigé (QTc) n'a pas été décrite. S'il s'agissait de la formule de Bazett (automatique), le QTc pourrait avoir été surestimé, et une correction par une autre formule (Fridericia) est à conseiller.
Malgré les quelques limites que nous venons de décrire, nous aimerions féliciter les auteurs d'avoir décrit un aspect clinique nécessitant une étude approfondie, qui pourrait s'avérer pertinent pour la prise en charge de la COVID-19. Il serait indiqué de mener d'autres recherches pour explorer et préciser la relation entre les lésions myocardiques et les issues négatives chez les patients hospitalisés en raison de la COVID-19.

\section{Références}

1. Calvillo-Argüelles O, Ross HJ. Cardiac considerations in patients with COVID19. CMAJ 2020;192:E630.

2. Driggin E, Madhavan MV, Bikdeli B, et al. Cardiovascular considerations for patients, health care workers, and health systems during the COVID-2019 pandemic. J Am Coll Cardiol 2020;75:2352-71.

3. Si D, Du B, Ni L, et al. Death, discharge and arrhythmias among patients with COVID-19 and cardiac injury. CMAJ 2020;192:E791-8.

4. Dhesi S, Shanks M, Tymchak WJ. Troponin rise in hospitalized patients with nonacute coronary syndrome: retrospective assessment of outcomes and predictors. Can J Cardiol 2015;31:296-301.

5. Poe S, Vandivier-Pletsch RH, Clay M, et al. Cardiac troponin measurement in the critically ill: potential for guiding clinical management. J Investig Med 2015;63:905-15.

6. Bessière F, Khenifer S, Dubourg J, et al. Prognostic value of troponins in sepsis: a meta-analysis. Intensive Care Med 2013;39:1181-9.

7. Lee KK, Noaman A, Vaswani A, et al. Prevalence, determinants, and clinical associations of high-sensitivity cardiac troponin in patients attending emergency departments. Am J Med 2019;132:110.e8-110.e21.

8. Lim W, Qushmaq I, Devereaux PJ, et al. Elevated cardiac troponin measurements in critically ill patients. Arch Intern Med 2006;166:2446-54.

9. Yu C-M, Wong RS-M, Wu EB, et al. Cardiovascular complications of severe acute respiratory syndrome. Postgrad Med J 2006;82:140-4.

Intérêts concurrents : Aucun déclaré.

Cet article a été sollicité et il n'a pas été révisé par des pairs.

Affiliations : Département de cardiologie, Université Queen's, Kingston, Ontario.

Collaborateurs : Les deux auteurs ont contribué à la conception du travail, ont rédigé le manuscrit et en ont révisé de façon critique le contenu intellectuel important; ils ont donné leur approbation finale pour la version destinée à être publiée et assument l'entière responsabilité de tous les aspects du travail.

Correspondance : Adrian Baranchuk, Adrian.Baranchuk@kingstonhsc.ca 